\title{
Correlations between Glass Structure and Emission Properties of Sn-Doped Zinc Phosphate Glasses Prepared with Different Cooling Rates
}

\author{
Aya Torimoto, ${ }^{*}$ Hirokazu Masai, ${ }^{1}$ Go Okada, ${ }^{2}$ \\ Takayuki Yanagida, ${ }^{2}$ and Masanori Koshimizu ${ }^{3}$ \\ Institute for Chemical Research, Kyoto University, Gokasho, Uji, Kyoto 611-0011, Japan \\ ${ }^{1}$ Department of Materials and Chemistry, National Institute of Advanced Industrial Science and Technology, \\ 1-8-31 Midorigaoka, Ikeda, Osaka 563-8577, Japan \\ ${ }^{2}$ Graduate School of Materials Science, Nara Institute of Science and Technology, \\ 8916-5 Takayama, Ikoma, Nara 630-0192, Japan \\ ${ }^{3}$ Department of Applied Chemistry, Graduate School of Engineering, Tohoku University, \\ Sendai, Miyagi 980-8579, Japan
}

(Received January 20, 2017; accepted July 28, 2017)

Keywords: phosphate glass, Sn, emission properties, structures, cooling rate

Photoluminescence (PL) and radiation-induced luminescence properties of Sn-doped zinc phosphate (SZP) glasses prepared with different cooling rates are investigated and associated with their glass structures. Although the shape of the PL spectrum is independent of the cooling rate, the emission intensity effectively changes with the cooling rate. The radial distribution functions of Sn in the SZP glasses prepared with different cooling rates coincide with each other. Electron spin resonance (ESR) measurement supports the fact that the trap density of slowly cooled glasses is higher than that of rapidly cooled glasses, and this fact is also suggested by the radiation-induced luminescence properties. Therefore, it is expected that these traps will work as storage sites associated with the radiation-induced luminescence.

\section{Introduction}

A melt quenching method is one of the most common methods for preparing glasses. In this method, a glass melt at a high temperature is continuously cooled down without crystallization at its melting temperature to convert it into the supercooled liquid state, and then frozen into the glassy state. This temperature has been defined as fictive temperature by Tool. ${ }^{(1)}$ Fictive temperature has been regarded as a parameter reflecting random structures; in other words, glasses exhibiting different fictive temperatures show different physical and chemical properties such as mechanical strength. ${ }^{(1,2)}$ Stebbins et al. have revealed that the $\mathrm{CaAl}_{2} \mathrm{Si}_{2} \mathrm{O}_{8}$ glass prepared with a high quenching rate consists of high concentrations of nonbridging oxygens and five-coordinated Al. ${ }^{(3)}$ They have also reported that the coordination number of boron atoms changes depending on the cooling rate in other aluminoborosilicate glasses. ${ }^{(4,5)}$ To the best of our knowledge, the correlation between the structure prepared with different cooling rates and the emission properties of the doped activators has rarely been investigated. The aggregation of $\mathrm{Sn}^{2+}$ is more likely to be

*Corresponding author: e-mail: torimotoaya0830@gmail.com

http://dx.doi.org/10.18494/SAM.2017.1617 
induced in the $\mathrm{SrO}-\mathrm{B}_{2} \mathrm{O}_{3}$ glasses prepared with low cooling rates. ${ }^{(6)}$ It goes without saying that glass structural properties, for example, the ratio of 3-coordinated borons/4-coordinated borons, the free volume, and so on, depend on the cooling rate. Consequently, there is a correlation among the cooling rate in the synthesis, glass structure, and emission properties.

In this paper, we focus on $\mathrm{Sn}$-doped $\mathrm{ZnO}-\mathrm{P}_{2} \mathrm{O}_{5}(\mathrm{SZP})$ glasses because their composition is well investigated. ${ }^{(7-12)} \mathrm{Sn}^{2+}$ centers belong to the group of $\mathrm{ns}^{2}$-type $(n \geq 4)$ emission centers, ${ }^{(13)}$ whose electrons in the outermost shell contribute to the emission process. This means that the emission properties are strongly affected by the coordination states of $\mathrm{ns}^{2}$-type emission centers. As reported in a previous paper, the quantum yield (QY) of SZP glasses is as high as that of rareearth ion-doped glasses and $\mathrm{MgWO}_{4}{ }^{(10)}$ Furthermore, it has been revealed that the melting in $\mathrm{Ar}$ atmosphere is effective in suppressing the oxidation of $\mathrm{Sn}^{2+}$ to $\mathrm{Sn}^{4+}$, and hence QY increases. ${ }^{(10,11,14,15)}$ Photoluminescence (PL) properties are related to the local structure of $\mathrm{Sn}^{2+}$; in contrast, radiationinduced luminescence properties are affected by both the host and local structures of $\mathrm{Sn}^{2+}$ centers. This is because the energy transfer from the host matrix to the emission centers occurs only in the radiation-induced luminescence process. Thus far, many researchers have reported on persistent luminescence and afterglow luminescence materials regardless of host materials. ${ }^{(16-18)}$ Thermally stimulated luminescence (TSL) and optically stimulated luminescence (OSL) properties were thoroughly investigated in order to discuss the energy levels and the density of traps. ${ }^{(16-18)}$ In a previous study, it was demonstrated that the addition of carbon to a glass batch is effective in obtaining a reducing atmosphere during the melting process; therefore, the reduced state of emission centers is increased. ${ }^{(19)}$ Moreover, it has been reported that the number of defect sites are increased in glasses and crystal systems. ${ }^{(20,21)}$ We assume that traps are an important factor of radiation-induced luminescence, especially in storage luminescence (TSL and OSL). Therefore, we believe that further investigation of such correlations between radiation-induced luminescence properties and the glass structure should be helpful in designing novel optical materials, scintillators, and dosimeters.

The objective of this study is to examine the correlation between the glass structure and the PL and radiation-induced luminescence properties of SZP glasses prepared with different cooling rates. To develop superior devices for X-ray detection (i.e., scintillators and dosimeters), much research has been intensively conducted. ${ }^{(22-26)}$

\section{Methods}

\subsection{Sample preparation}

Hereinafter, the glasses of $x \mathrm{SnO}-60 \mathrm{ZnO}-40 \mathrm{P}_{2} \mathrm{O}_{5}(x=0,0.1,0.5$, and $1.0 \mathrm{~mol} \%)$ prepared with low and high cooling rates are denoted as " $x \mathrm{SZP}: l$ " and " $x \mathrm{SZP}: h$ ", respectively. First, $\mathrm{ZnO}$ and $\left(\mathrm{NH}_{4}\right)_{2} \mathrm{HPO}_{4}$ were mixed and calcined according to a previous paper. ${ }^{(12)}$ Second, a stoichiometric amount of $\mathrm{SnO}$ was added to the powdered calcined sample. The conventional melt quenching method was employed to prepare the SZP glass with a high cooling rate, according to a previous paper. ${ }^{(12)}$ The heating program of the glasses prepared with a low cooling rate is as follows: (1) The mixed sample was heated from room temperature (r.t.) to $1100{ }^{\circ} \mathrm{C}$ for $3 \mathrm{~h}$. (2) The temperature was kept at $1100{ }^{\circ} \mathrm{C}$ for $1 \mathrm{~h}$ in Ar atmosphere. (3) The sample was cooled from $1100{ }^{\circ} \mathrm{C}$ to r.t. for 3 h. Then, both glasses were cut into pieces with dimensions of $10 \times 10 \times 1 \mathrm{~mm}^{3}$ and mechanically polished to obtain a mirror plane. 


\subsection{Analysis}

$T_{g}$ was examined by DTA using Thermo Plus 8120 (Rigaku) at a heating rate of $10{ }^{\circ} \mathrm{C} / \mathrm{min}$. Densities were determined by using an Archimedes method with distilled water as the immersion liquid at r.t. PL and PL excitation (PLE) spectra were recorded on a fluorescence spectrophotometer (F-7000, Hitachi). Slits for achieving an optical resolution of $2.5 \mathrm{~mm}$ were used for excitation and emission measurements. QY was measured using Quantaurus-QY (Hamamatsu Photonics). PL decay profiles at r.t. were conducted on Quantaurus-Tau (Hamamatsu Photonics) with a $280 \mathrm{~nm}$ LED source. Sn K-edge (29.3 keV) extended X-ray absorption fine structure (EXAFS) spectra were recorded at BL14B02 of SPring-8 (Hyogo, Japan). The storage ring energy source was operated at $8 \mathrm{GeV}$ with a typical current of $100 \mathrm{~mA}$. The measurements were carried out using a $\mathrm{Si}$ (311) double-crystal monochromator in the transmission mode (Quick Scan method) at r.t. X-ray scintillation spectra at r.t. were obtained with a monochromator equipped with a charge-coupled device (CCD, Andor DU-420-BU2). The irradiated dose was calibrated using an ionization chamber. OSL spectra were recorded by Quantaurus-Tau (Hamamatsu Photonics) and the stimulation wavelength of light was $630 \mathrm{~nm}$, equivalent to $1.97 \mathrm{eV}$. TSL glow curves were recorded using TL-2000 (Nano Gray). Photons over $500 \mathrm{~nm}$ were cut using a thermal radiation cut filter and the photomultiplier tube accurately detects photons above approximately $300 \mathrm{~nm}$; therefore, the spectral range was from 300 to $500 \mathrm{~nm}$. The temperature range of the TSL measurement was from 50 to $400{ }^{\circ} \mathrm{C}$. All samples were measured immediately after $10 \mathrm{~Gy}(40 \mathrm{kV}, 5.2 \mathrm{~mA}, 10 \mathrm{~min})$ irradiation in the same manner as X-ray-induced scintillation spectra. Electron spin resonance (ESR) spectra were obtained using an ESR spectrometer (JES X330, JEOL). The modulation width and microwave power were $0.5 \mathrm{mT}$ and $160 \mathrm{~mW}$, respectively.

\section{Results and Discussion}

As shown in Fig. 1 (left axis), the $x \mathrm{SZP}: h\left(x=0,0.1,0.5\right.$, and 1.0) glass showed a higher $T_{g}$ than the $x \mathrm{SZP}: l(x=0,0.1,0.5$, and 1.0) glass, respectively. This result agrees with the conventional tendency: a high cooling rate gives a high fictive temperature. ${ }^{(1)} T_{g}$ decreases with increasing amount of $\mathrm{SnO}$ regardless of the different cooling rates. The densities of all samples are also exhibited in Fig. 1 (right axis). The rapidly cooled glasses have a lower density than the slowly cooled glasses, indicating that a larger free volume exists in the former glasses. This relationship between the density and the cooling rate is also in good agreement with that in a previous paper. ${ }^{(27)}$

To discuss more quantitatively, the normalized PL-PLE spectra are presented in Fig. 2 and the relative PL intensities are described in parentheses. A high PL intensity is observed in the rapidly cooled glass, in the order 1.0SZP: $h>0.5 \mathrm{SZP}: h>0.1 \mathrm{SZP}: h$. For the slowly cooled glass, the order of the PL intensity observed is $0.5 \mathrm{SZP}: l>1.0 \mathrm{SZP}: l>0.1 \mathrm{SZP}: l$. The spectral shapes of the glasses with the same chemical composition are almost the same even though they are prepared with different cooling rates.

PL decay profiles of all samples monitored with $280 \mathrm{~nm}$ excitation are presented in Fig. 3. The decay profiles exhibit linearity, therefore indicating that only a single component exists. Furthermore, the decay constants accord with the $\mathrm{Sn}^{2+}$ centers. ${ }^{(7-9,11,13,29)}$ Considering the PL spectra and decay curves, the radiative process is almost single, although there exist two different excitation states of $\mathrm{Sn}^{2+}$. Figure 4 shows the QY of both $x \mathrm{SZP}: l$ and $x \mathrm{SZP}: h$ glasses. The observed tendency is in good agreement with the order of the emission intensity detected in PL-PLE spectra. 


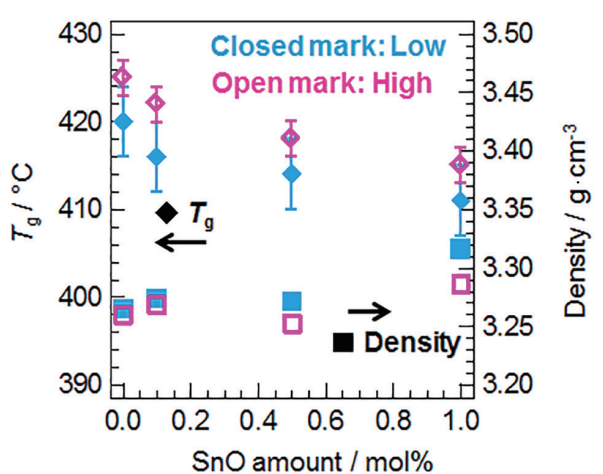

Fig. 1. (Color online) $T_{g}$ 's (left axis) and densities (right axis) of $x \operatorname{SZP}(x=0,0.1,0.5$, and 1.0) glass prepared with different cooling rates.

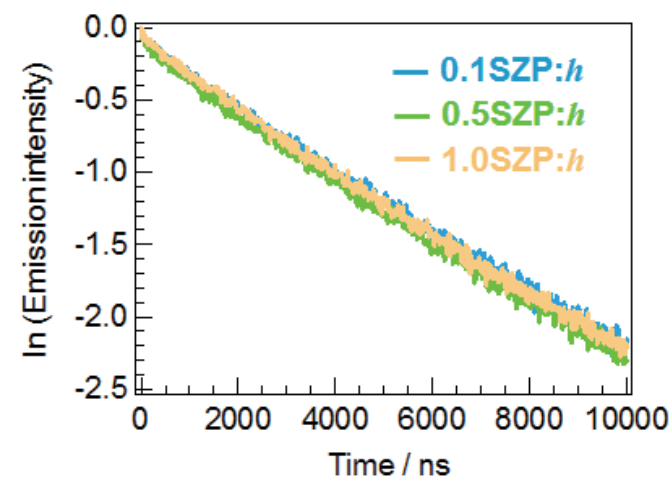

(a)

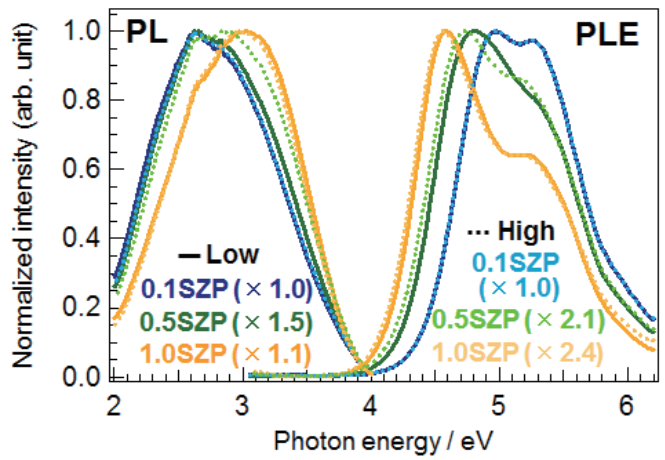

Fig. 2. (Color online) Normalized PL-PLE spectra of $x \mathrm{SZP}(x=0.1,0.5$, and 1.0$)$ glass prepared with different cooling rates.

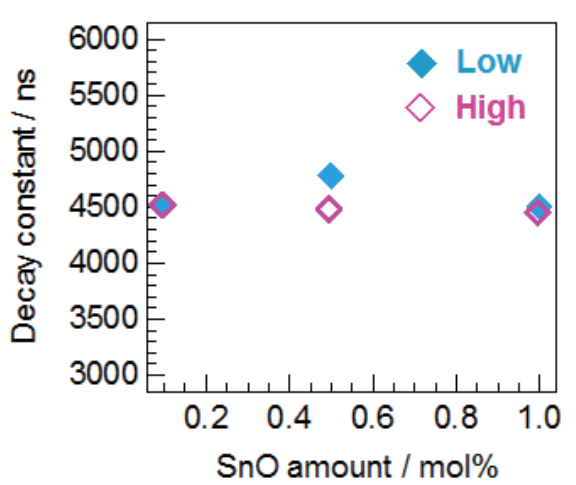

(b)

Fig. 3. (Color online) PL decay profiles of $x \mathrm{SZP}: h(x=0.1,0.5$, and 1.0) glass. (b) Decay constants of $x \mathrm{SZP}(x=$ $0.1,0.5$, and 1.0) glass prepared with different cooling rates as a function of $\mathrm{SnO}$ amount.

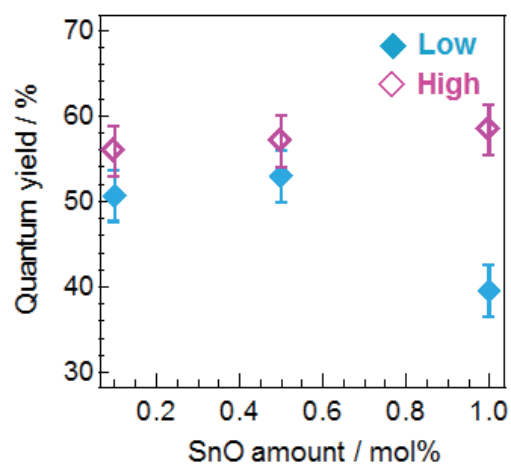

Fig. 4. (Color online) QY of $x \operatorname{SZP}(x=0.1,0.5$, and 1.0) glass prepared with different cooling rates.

Judging from the PL properties discussed above, it is predicted that the local structure of $\mathrm{Sn}^{2+}$ centers will not be affected by the cooling rate. We, therefore, conducted EXAFS measurement in order to investigate the local structure of $\mathrm{Sn}^{2+}$. Fourier transforms of EXAFS spectra, equivalent 
to radial distribution functions, of $\mathrm{Sn}$ in the 1.0SZP glasses prepared with different cooling rates were in good agreement as shown in Fig. 5. This confirms that the first coordination sphere remains unchanged even if the cooling rate of the glass differs. Consequently, both PL property surveys and EXAFS spectra affirm that the local structure of $\mathrm{Sn}^{2+}$ centers is independent of the cooling rate in the SZP glass.

Figure 6(a) shows the X-ray scintillation spectra of the $0.5 \mathrm{SZP}: h$ glass irradiated under different $\mathrm{X}$-ray doses (0.01-10 Gy) as a representative sample. Figure 6(b) presents the emission intensities of all samples as a function of irradiation dose. There is good linearity depending on the $\mathrm{Sn}^{2+}$ concentration. The most notable point is that rapidly cooled glasses tend to show a higher emission intensity than slowly cooled glasses as long as the same amount of $\mathrm{Sn}$ is added. This indicates that a larger number of trap sites exist in slowly cooled glasses.

The OSL spectra of the $x$ SZP: $l(x=0,0.1,0.5$, and 1.0) glass and the OSL intensities of all samples as a function of the amount of $\mathrm{SnO}$ are denoted in Figs. 7(a) and 7(b), respectively. We measured the OSL spectra by 630 and $590 \mathrm{~nm}$ stimulations, and confirmed that no significant difference is observed between them. We therefore assumed that $630 \mathrm{~nm}$ stimulation is enough

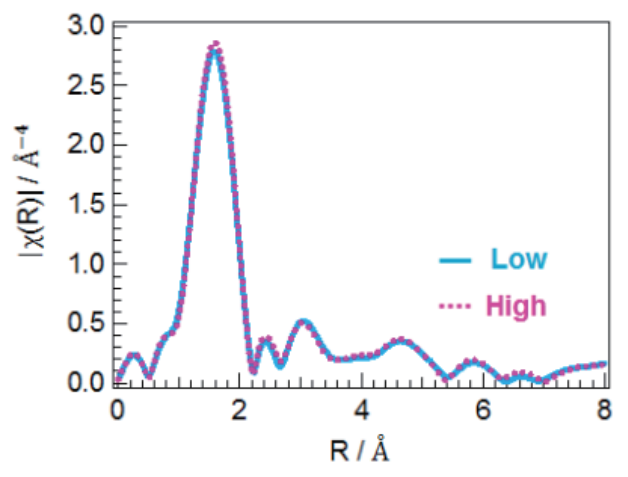

Fig. 5. (Color online) Radial distribution functions of $\mathrm{Sn}$ in 1.0SZP glasses prepared with different cooling rates.

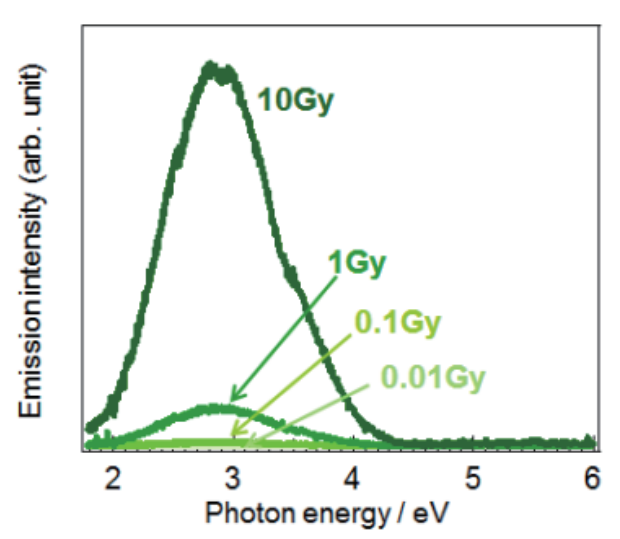

(a)

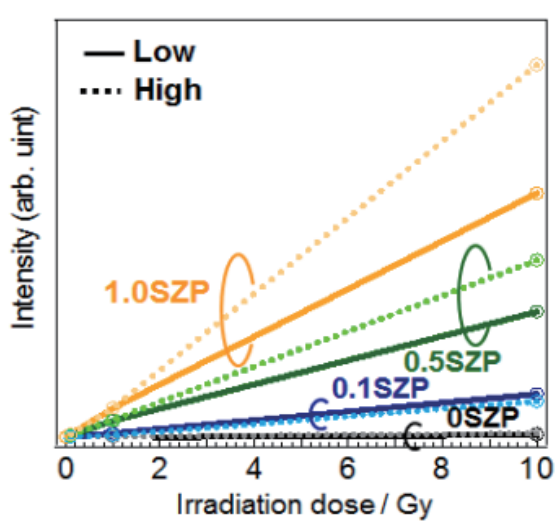

(b)

Fig. 6. (Color online) (a) X-ray-induced scintillation spectra of 0.5SZP: $h$ glass as a representative. (b) Emission intensity of $x$ SZP glasses $(x=0,0.1,0.5$, and 1.0) as a function of irradiation dose. 


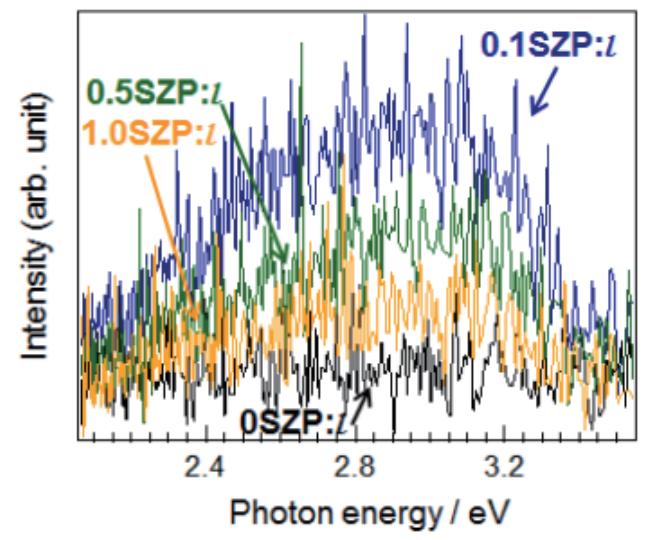

(a)

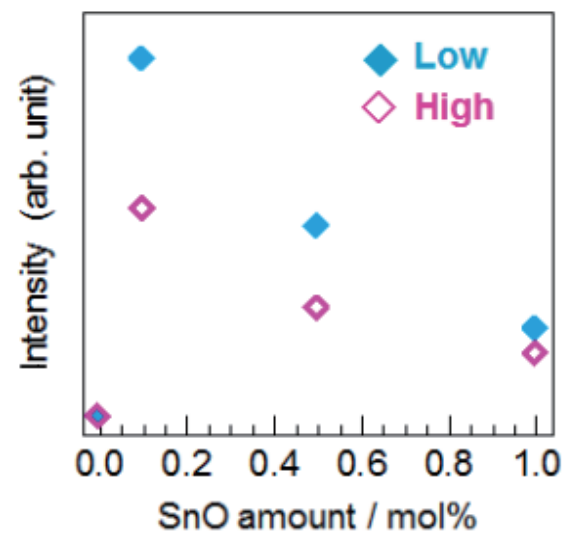

(b)

Fig. 7. (Color online) (a) OSL spectra of $x \mathrm{SZP}: l(x=0,0.1,0.5$, and 1.0) glass after 10 Gy radiation. (b) Emission intensities of $x \operatorname{SZP}(x=0,0.1,0.5$, and 1.0) glass prepared with different cooling rates as a function of $\mathrm{SnO}$ amount.

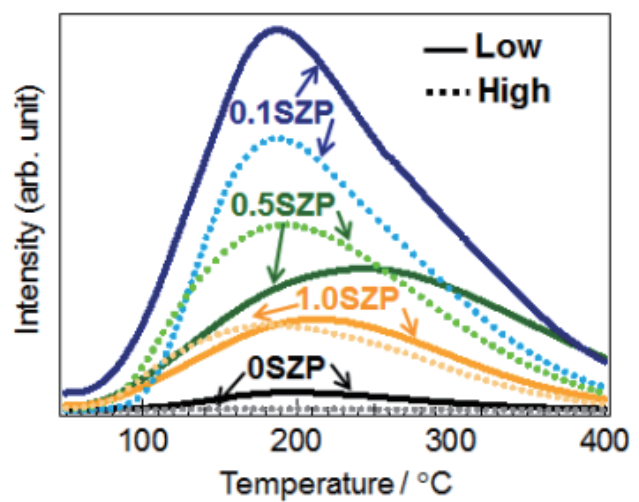

(a)

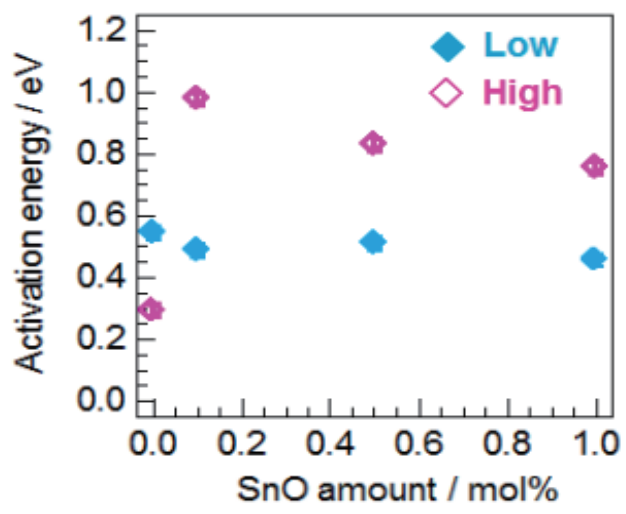

(b)

Fig. 8. (Color online) (a) TSL glow curves of $x \operatorname{SZP}(x=0,0.1,0.5$, and 1.0) glass prepared with different cooling rates. The irradiation dose was $10 \mathrm{~Gy}$. (b) Activation energies of $x \operatorname{SZP}(x=0,0.1,0.5$, and 1.0) glass prepared with different cooling rates as a function of $\mathrm{SnO}$ amount.

for the release of the storage energy, and selected the wavelength of $630 \mathrm{~nm}$ for the stimulation. The slowly cooled glasses show relatively higher emission intensities than the rapidly cooled glasses. Therefore, it is suggested that the number density of traps storing electrons is higher, which coincides with the discussion in X-ray scintillation spectra. More interestingly, the order of intensity is as follows; the amount of $\mathrm{SnO}$ is $0.1>0.5>1.0 \mathrm{~mol} \%$. It means that the number density of traps changes with the concentration of $\mathrm{Sn}^{2+}$ centers. The order of the emission intensity of the TSL glow curves of all $x \operatorname{SZP}(x=0,0.1,0.5$, and 1.0) glasses [Fig. 8(a)] is the same as that in OSL spectra in terms of the cooling rate and the amount of SnO. The activation energy (i.e., thermal energy required to liberate a trapped electron) determined by the initial rise $\operatorname{method}^{(30)}$ is plotted as a function of $\mathrm{SnO}$ amount in Fig. 8(b). The energy levels of traps in the slowly cooled glasses are lower than those in the rapidly cooled glass. 


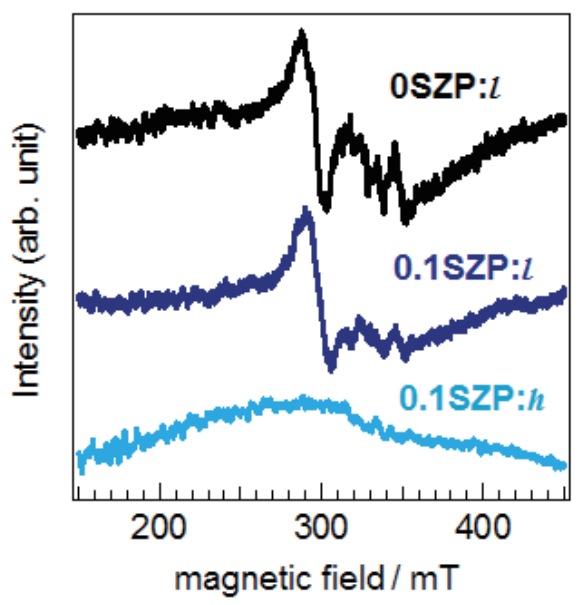

Fig. 9. (Color online) ESR spectra of $x \mathrm{SZP}: l(x=0$ and 0.1$)$ and 0.1SZP: $h$ glasses.

It is considered that the host structure depends on the cooling rate on the basis of the radiationinduced luminescence properties. No significant difference was observed in both ${ }^{31} \mathrm{P}$ MAS NMR and IR spectra. In the ESR spectra (Fig. 9), however, a notable difference was detected between the slowly and rapidly cooled glasses. Signals are found in the slowly cooled glasses, 0SZP:l and 0.1SZP:l. We attribute the origin of the trap sites that induce the higher emission intensity in storage luminescence to some unpaired electron species such as $\mathrm{P}-\dot{\mathrm{O}}$ and $\mathrm{Zn}-\dot{\mathrm{O}}$. These unpaired electron species are perhaps due to the different cooling rates or materials originating from the crucible (i.e., Pt or glassy carbon).

\section{Conclusions}

In this study, we have investigated PL and radiation-induced luminescence properties of SZP glasses prepared with different cooling rates, and correlated these properties with the glass structures. The PL intensity depends on the cooling rate, whereas the spectral shapes of PL-PLE spectra are independent of the cooling rate. The Sn K-edge EXAFS measurement reveals that the first coordination spheres are similar despite the difference in cooling rate. On the other hand, the radiation-induced luminescence properties suggest that a larger number of traps, whose role is to store electrons and/or holes, is generated in the slowly cooled glasses than in the rapidly cooled glasses. The ESR measurement confirms that ESR-active defects are generated more effectively in the slowly cooled glasses than in the rapidly cooled glasses. The relationship of cooling rate with the storage luminescence properties should be studied in detail in future works.

\section{Acknowledgments}

This work was partially supported by the JSPS KAKENHI Grant-in-Aid for Young Scientists (A) (Grant No. 26709048), the Izumi Science and Technology Foundation, the Collaborative Research Program of Institute for Chemical Research, Kyoto University (Grant No. \#2016-83), and the Cooperative Research Project of the Research Institute of Electronics, Shizuoka University. The synchrotron radiation experiments were performed at the BL14B2 of SPring- 8 with the approval of the Japan Synchrotron Radiation Research Institute (JASRI) (Proposal No. 2015B1587). We thank Dr. H. Ofuchi for supporting the EXAFS measurements at the BL14B2. 


\section{References}

1 Y. Yue, R. von der Ohe, and S. L. Jensen: J. Chem. Phys. 120 (2004) 8053.

2 A. Koike and M. Tomozawa: J. Non-Cryst. Solids 352 (2006) 5522.

3 J. F. Stebbins, E. V. Dubinsky, K. Kanehashi, and K. E. Kelsey: Geochim. Cosmochim. Acta. 72 (2008) 910.

4 J. F. Stebbins and S. E. Ellsworth: J. Am. Ceram. Soc. 79 (1996) 2247.

5 J. Wu and J. F. Stebbins: J. Non-Cryst. Solids 356 (2010) 2097.

6 H. Masai, A. Koreeda, Y. Fujii, T. Ohkubo, and S. Kohara: Opt. Mater. Express 6 (2016) 1827

7 A. Torimoto, H. Masai, Y. Tokuda, T. Yanagida, G. Okada, and K. Mibu: J. Ceram. Soc. Jpn. 124 (2016) 554.

8 A. Torimoto, H. Masai, G. Okada, and T. Yanagida: Radiat. Meas. (in press).

9 H. Masai, T. Tanimoto, T. Fujiwara, S. Matsumoto, Y. Tokuda, and T. Yoko: Opt. Express 20 (2012) 27319.

10 H. Masai, Y. Takahashi, T. Fujiwara, S. Matsumoto, and T. Yoko: Appl. Phys. Express 3 (2010) 082102.

11 H. Masai, T. Tanimoto, S. Okumura, K. Teramura, S. Matsumoto, T. Yanagida, Y. Tokuda, and T. Yoko: J. Mater. Chem. C 2 (2014) 2137.

12 H. Masai, T. Tanimoto, T. Fujiwara, S. Matsumoto, Y. Takahashi, Y. Tokuda, and T. Yoko: J. Non-Cryst. Solids 358 (2012) 265.

13 W. M. Yen, S. Shionoya, and H. Yamamoto: Phosphor Handbook 2nd Edition (CRC Press, Boca Raton, 2007).

14 T. Akai: New Glass 22 (2007) 54 (in Japanese).

15 H. Segawa, S. Inoue, and K. Nomura: J. Non-Cryst. Solids 358 (2012) 1333.

16 P. Li, M. Peng, L. Wondraczek, Y. Zhaoa, and B. Vianac: J. Mater. Chem. C 3 (2015) 3406.

17 N. Da, M. Peng, S. Krolikowski, and L. Wondraczek: Opt. Express 18 (2010) 2550.

18 Yixi Zhuang, J. Ueda, and S. Tanabe: J. Mater. Chem. C 1 (2013) 7849.

19 A. D. Sontakke, J. Ueda, and S. Tanabe: J. Non-Cryst. Solids 431 (2016) 150.

20 H. Masai, K. Hamaguchi, K. Iwasaki, Y. Takahashi, R. Ihara, and T. Fujiwara: Mater. Res. Bull. 47 (2012) 4065.

21 K. Palanivelu, J. S. Im, and Y. S. Lee: Carbon Sci. 8 (2007) 214.

22 M. Ishii, Y. Kuwano, T. Asai, S. Asaba, M. Kawamura, N. Senguttuvan, T. Hayashi, M. Kobayashi, M. Nikl, S. Hosoya, K. Sakai, T. Adachi, T. Oku, and H. M. Shimizu: Nucl. Instrum. Methods Phys. Res., Sect. A 537 (2005) 282.

23 H. Tatsumi, G. Okada, T. Yanagida, and H. Masai: J. Ceram. Soc. Jpn. 124 (2016) 550.

24 Y. Miyamoto, T. Ohno, Y. Takei, H. Nanto, T. Kurobori, T. Yanagida, A. Yoshikawa, Y. Nagashima, and T. Yamamoto: Radiat. Meas. 55 (2013) 72.

25 A. Timar-Gabor, C. Ivascu, S. Vasiliniuc, L. Daraban, I. Ardelean, C. Cosma, and O. Cozar: Appl. Radiat. Isot. 69 (2011) 780.

26 C. A. G. Kalnins, H. Ebendorff-Heidepriem, N. A. Spooner, and T. M. Monro1: Opt. Mater. Express 2 (2012) 62.

27 M. D. Ediger, C. A. Angell, and S. R. Nagel: J. Phys. Chem. 100 (1996) 13200.

28 L. Skuja: J. Non-Cryst. Solid. 149 (1992) 77.

29 H. Masai, Y. Hino, T. Yanagida, Y. Fujimoto, K. Fukuda, and T. Yoko: J. Appl. Phys. 114 (2013) 083502.

30 C. S. Shalgaonkar and A. V. Narlikar: J. Mater. Sci. 7 (1972) 1465. 